\title{
Meaningfulness in visual complexity ${ }^{1}$
}

\author{
FRANK H. FARLEY, University of \\ Wisconsin, Madison, Wis. 53706
}

The meaningfulness (production $\mathrm{m}$ ) of random polygons varying in complexity by approximately even logarithmic steps from 4 to 160 sides, but matched in area, was studied. $A$ 60-sec exposure and production period was used with 52 Ss. The possibility raised by previous research that visual complexity and meaningfulness are significantly related was not supported.

The association value and meaningfulness of random shapes varying in complexity has been reported by Vanderplas \& Garvin (1959) and Eisenman (1966). The former authors presented polygons for $3 \mathrm{sec}$ each, with the $S$ requested to report any associations he had to the polygon or simply to report "yes" if the polygon reminded him of something he was unable to label. The polygons used were random shapes constructed by the Attneave \& Arnoult (1956) procedures, with complexity defined in terms of the number of sides. Six levels of complexity were used $(4,6,8,12,16$, and 24 sides). Two principal measures were obtained. One was simply the percentage of the $50 \mathrm{Ss}$ tested who reported an association or stated "yes" for a given figure, while the second measure was the content value of the responses given and was the total percentage of responses that were words or phrases denoting associations with objects or situations. The range of association values obtained was $20 \%$ to $62 \%$, with a mean of $38 \%$. Eisenman (1966) noted that the conditions of testing in the Vanderplas and Garvin study may have been such as to restrict the percentage of associations and content responses obtained, that is, darkened experimental room, onset and offset of the polygon presentations signaled by buzzer, and requirement of a spoken response. Eisenman presented $22 \mathrm{Ss}$ with polygons from the 4-, 12-, and 24-point categories of Vanderplas and Garvin, as well as three symmetrical shapes of 4,8 , and 10 points from Birkhoff (1933), and had Ss write their associations in a regular classroom setting, giving them as long as they wanted during a $1-\mathrm{h}, 40-\mathrm{min}$ class period. "However, Ss were requested to mark down their first association and not to spend too much time on the polygons [p. 397]." Eisenman's study yielded a significantly higher percentage of associations than those found by Vanderplas and Garvin for the same polygons. The percentage of content responses was also significantly greater in the Eisenman study.

Vanderplas and Garvin reported a trend toward lower associative value for more complex polygons. Eisenman did not obtain such a trend, reporting the highest associative value for the 12 -sided polygon of the 4-, 12-, and 24-sided polygons replicated from Vanderplas and Garvin. Munsinger \& Kessen (1964) used 48 Ss and a paired comparison procedure with random shapes of from 5 to 40 sides, asking Ss to report which one of a pair reminded them of more things, that is, which shape was more meaningful, presenting the pairs for $10 \mathrm{sec}$ each. These authors found that greater complexity was associated with greater meaningfulness. Finally, Evans \& Day (1968), employing polygons of three levels of complexity (10, 28 , and 80 sides), with each figure presented to $64 \mathrm{Ss}$ for $15 \mathrm{sec}$ and $\mathrm{S}$ requested to write down his associations, found that across the three complexity levels sampled, lesser association value was found with greater complexity, the opposite of the Munsinger and Kessen results but a similar result to that of Vanderplas and Garvin.

It can be seen that the procedures used in relevant studies have varied considerably, with the most notable variations being in the range of complexity sampled and the method of obtaining associations, particularly in the time allowed for the latter. Vanderplas and Garvin, Eisenman, and Evans and Day have all employed a meaningfulness measure somewhat like Noble's (1952) production " $m$," which has been widely used in obtaining meaningfulness data for verbal materials, but none have obtained the data under conditions directly comparable to the measurement of $m$ for verbal stimuli. The number of complexity levels sampled has been quite restricted in most of the studies. Clearly, any satisfactory evaluation of the possible relationship of meaningfulness to complexity would require utilization of a wide range of complexity levels.

Accordingly, the present study was undertaken to evaluate meaningfulness in relation to visual complexity, using a range of complexity values greater than has heretofore been employed in meaningfulness research and obtaining the meaningfulness data under conditions comparable to Noble's $m$.

\section{SUBJECTS}

Fifty-two university students (primarily undergraduate) from a class in human learning and abilities were tested. Participation in the experiment provided Ss with experimental credits that could be applied to their course grade if their course performance was borderline between two letter grades. Twenty-six were females and 26 were males.

\section{STIMULUUS MATERIALS AND PROCEDURE}

Twenty-two black-on-white polygons, varying in number of sides in approximately equal logarithmic steps from 4 to 160, were used (Day, 1967). They had been constructed using Attneave \& Arnoult's (1956) Method 1, with the figures equated in size and photographed on $35-\mathrm{mm}$ high-contrast film, being mounted on slides.

The polygons were presented individually by a Kodak Carousel projector to groups of approximately four to five $S s$ at a time, in a normally lighted room. Following Noble (1952), the stimulus figure was present for $60 \mathrm{sec}$, during which time $\mathrm{S}$ was to write down his discrete associations to the stimulus. Noble's instructions were used except for slight modifications due to the pictorial as opposed to the verbal nature of the stimuli. A given stimulus therefore was present throughout the period during which $S$ recorded his responses. At the termination of the $60 \mathrm{sec}$, the next polygon was presented, and the $S$ turned to a fresh page in his response booklet. Five different random orders of the polygons were used. The assignment of order to testing group was random.

RESULTS
Associations were scored using Noble's criteria. The total number of discrete associations was 3,297 . The mean number per figure was 2.882 . Interestingly, the proportion of instances in which no scorable response was provided for a given polygon was so low that the usual analysis of the percentage of Ss yielding an association for a given figure was precluded. (Out of 1,144 protocols, that is, 52 Ss $x 22$ polygons, only 63 provided no

Table 1

Mean Number of Associations to Polygons of 4-160 Sides

\begin{tabular}{lccc}
$\begin{array}{l}\text { Polygon } \\
\text { (Number }\end{array}$ & $\begin{array}{c}\text { Associa- } \\
\text { tions }\end{array}$ & $\begin{array}{l}\text { Polygon } \\
\text { (Number } \\
\text { of Sides) }\end{array}$ & $\begin{array}{c}\text { Associa- } \\
\text { tions }\end{array}$ \\
\hline 4 & 3.077 & 46 & 2.942 \\
6 & 2.846 & 54 & 2.885 \\
10 & 3.442 & 62 & 2.442 \\
12 & 2.692 & 70 & 2.712 \\
14 & 2.327 & 80 & 3.019 \\
16 & 3.058 & 90 & 2.885 \\
20 & 2.596 & 100 & 2.712 \\
24 & 2.923 & 122 & 2.923 \\
28 & 2.750 & 134 & 2.904 \\
34 & 2.981 & 146 & 3.481 \\
40 & 2.712 & 160 & 3.096 \\
\hline
\end{tabular}


association.) Accordingly, the mean number of associations per figure was computed and used as the index of meaningfulness. The results of this analysis are found in Table 1. From Table 1 it can be seen that no clear trend of relationship of complexity to production $m$ has been obtained. In order to estimate the strength of the relationship, a Pearson correlation was computed, yielding a nonsignificant value of .27 .

\section{DISCUSSION}

The present meaningfulness data, using a close approximation to Noble's technique and the widest range of complexity values yet employed on this topic, has clearly indicated no significant relationship of meaningfulness to complexity. However, variability in meaningfulness has been obtained; the factors that elicit this variability have not been identified here. The lack of relationship of complexity to meaningfulness is particularly interesting in light of the conflicting literature. Two studies have reported lesser meaningfulness for more complex figures (Evans \& Day, 1968; Vanderplas \& Garvin, 1959), but both used short exposure times (15 sec and $3 \mathrm{sec}$, respectively) and both included few levels of complexity ( 3 and 6, respectively, compared to the present 22). Munsinger \& Kessen (1964) reported the opposite result, that is, greater meaningfulness for more complex figures. They used a $10-\mathrm{sec}$ exposure duration and a paired-comparisons technique. However, no actual associative productions were requested of S. Eisenman (1966), using the 4-, 12-, and 24-sided figures from Vanderplas and Garvin, with "unlimited time" for the production of associations, found the middle figure, 12 , to have the highest associative value. On the other hand, the latter authors found that both their 4- and 6-sided figures more often produced high associative values than did the 12-sided one.

The Munsinger and Kessen results are not very readily comparable with the remaining studies because of the important differences in procedure. However, the remaining studies seem primarily differentiated on the temporal variable, that is, the time allowed to produce associations to the stimuli. When relatively brief durations were used (Evans \& Day, Vanderplas \& Garvin), a negative relationship of associative value to complexity was obtained. When a long duration was used (Eisenman), middle values of complexity were most meaningful. However, the latter study indicated that the 12-sided polygon was most meaningful; it should be noted that the simplest polygon employed by Evans and Day had 10 sides and had the highest

associative value. On the other hand, the present 4-, 12-, and 24-sided figures produced the lowest associative value for 12 with the highest for 4 and 24, respectively. Five of the present figures (4, $6,12,16,24)$ replicated the complexity levels reported by Vanderplas and Garvin. However, inspection of the two sets of results indicates that the complexity-association value relationship was not replicated.

The trend of results thus far suggest that the relationship of associative value to complexity may depend on the length of time the $S$ views the figure. It might be hypothesized that, as exposure duration increases from very brief periods, the relationship of complexity to meaningfulness changes from negative to positive. Clearly, research should be undertaken which systematically varies the temporal parameters. It would be advised that such research include a wide range of complexity levels with a number of examples at each level.

On the basis of the present study, however, which has attempted to duplicate procedures widely used in meaningfulness studies with verbal stimuli, it must be concluded that no significant relationship between visual complexity and meaningfulness has been obtained.

\section{Effects of syntax on running memory span for connected discourse}

ROBERT J. JARVELLA, ${ }^{1}$ Program in Psycholinguistics, University of Michigan, Ann Arbor, Mich. 48104

Running memory span (RMS) was tested during pauses placed at word boundaries in connected discourse. RMS for the seven words previous to test pauses was superior when all seven fell into the same clause, as opposed to when only the last four words were from the immediate clause. The results gave some support to a hypothesis made about syntactic structure in immediate memory, and their relation to previous findings on speech segmentation in listening was discussed.

Studies on the segmentation of

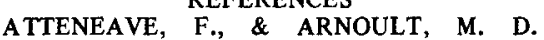
Methodological considerations in the quantitative study of shape and pattern in perception. Psychological Bulletin, 1956, 53, 452-471.

BIRKHOFF, G. D. Aesthetic measure. Cambridge: Harvard University Press, 1933.

DAY, H. I. Evaluations of subjective complexity, pleasingness and interestingness for a series of random polygons varying in complexity. Perception \& Psychophysics, 1967, 2, 281-286.

EISENMAN, R. The association value of random shapes revisited. Psychonomic Science, 1966, 6, 397-398.

EVANS, D. R., \& DAY, H. I. Association value and subjective ratings of interest in visual complexity. Paper presented at American Educational Research Association Annual Meeting, Chicago, February 1968.

MUNSINGER, H., \& KESSEN, W. Uncertainty, structure, and preference. Psychological Monographs, 1964, 78, 1-24.

NOBLE, C. E. An analysis of meaning. Psychological Review, 1952, 59, 421-430.

VANDERPLAS, J. M., \& GARVIN, E. A. The association value of random shapes. Journal of Experimental Psychology, 1959, 57, 147-154.

NOTE

1. The author would like to thank Mrs. Sue Green for assistance in conducting the study. This research was supported in part by the United States Office of Education, Department of Health, Education and Welfare, Center No. C-03/Contract OE 5-10-154. sentences into phrases during speech processing have shown that clicks located acoustically within sentences are displaced toward clause boundaries in listeners' later subjective locations (Fodor \& Bever, 1965 ; Garrett, Bever, \& Fodor, 1966). This finding has been interpreted as evidence that clause structure is actively imposed on the speech signal during listening. The errors made in locating clicks in sentences are considered to be a function of interference with attention to competing sounds during perceptual analysis of the immediate speech stimulus.

In the present study, a similar syntactic effect on the processing of continuous speech was evaluated in a recall task and related to a hypothesis about structural 